\title{
High-Throughput Computing on High-Performance Platforms: A Case Study
}

\author{
Danila Oleynik* , Sergey Panitkin*§, Matteo Turilli*†, \\ Alessio Angius ${ }^{\dagger}$, Sarp Oral ${ }^{\|}$, Kaushik De ${ }^{\Uparrow}$, Alexei Klimentov ${ }^{\S}$, Jack C. Wells** and Shantenu Jha ${ }^{\dagger \ddagger}$ \\ ${ }^{*}$ First authors, alphabetical order \\ ${ }^{\dagger}$ Department of Electric and Computer Engineering, Rutgers University, NJ, USA \\ $\ddagger$ Computational Science Initiative, Brookhaven National Laboratory, NY, USA \\ $\S$ Physics Department, Brookhaven National Laboratory, NY, USA \\ TPhysics Department, University of Texas Arlington, TX, USA \\ | Oak Ridge Leadership Computing Facility, Oak Ridge National Laboratory, TN, USA \\ **National Center for Computational Sciences, Oak Ridge National Laboratory, TN, USA
}

\begin{abstract}
The computing systems used by LHC experiments has historically consisted of the federation of hundreds to thousands of distributed resources, ranging from small to mid-size resource. In spite of the impressive scale of the existing distributed computing solutions, the federation of small to mid-size resources will be insufficient to meet projected future demands. This paper is a case study of how the ATLAS experiment has embraced Titan-a DOE leadership facility in conjunction with traditional distributed high- throughput computing to reach sustained production scales of approximately $52 \mathrm{M}$ core-hours a years. The three main contributions of this paper are: (i) a critical evaluation of design and operational considerations to support the sustained, scalable and production usage of Titan; (ii) a preliminary characterization of a next generation executor for PanDA to support new workloads and advanced execution modes; and (iii) early lessons for how current and future experimental and observational systems can be integrated with production supercomputers and other platforms in a general and extensible manner.
\end{abstract}

\section{INTRODUCTION}

The Large Hadron Collider (LHC) was created to explore the fundamental properties of matter. Multiple experiments at LHC have collected and distributed hundreds of petabytes of data worldwide to hundreds of computer centers. Thousands of physicists analyze petascale data volumes daily. The detection of the Higgs Boson in 2013 speaks to the success of the detector and experiment design, as well as the sophistication of computing systems devised to analyze the data, which historically, consisted of the federation of hundreds to thousands of distributed resources, ranging in scale from small to mid-size resource [1].

The LHC workloads are comprised of tasks that are independent of each other, however, the management of the distribution of workloads across many heterogeneous resources, the effective utilization of resources and efficient execution of workloads present non-trivial challenges. Many software solutions have been developed in response to these challenges. The CMS experiment, devised a solution based around the HTCondor [2] software ecosystem. The ATLAS [3] experiment utilizes the Production and Distributed Analysis (PanDA) workload management system [4] (WMS) for dis- tributed data processing and analysis. The CMS and ATLAS experiments utilize, arguably the largest academic production grade distributed computing solutions, and have symbolized the paradigm of high-throughput computing (HTC), i.e., the effective execution of many independent tasks.

In spite of the impressive scale of the ATLAS distributed computing system-in the number of tasks executed, the number of core hours utilized, and the number of distributed sites utilized, demand for computing systems will soon significantly outstrip current and projected supply. The data volumes that will need analyzing in LHC-Run $3(\approx 2022)$ and the highluminosity era (Run 4) will increase by factors of 10-100 compared to the current phase (Run 2). There are multiple levels at which this problem needs to be addressed: the utilization of emerging parallel architectures (e.g., platforms); algorithmic and advances in analytical methods (e.g., use of Machine Learning); and the ability to exploit different platforms (e.g., clouds and supercomputers).

This paper represents the experience of how the ATLAS experiment has "broken free" of the traditional computational approach of high-throughput computing on distributed resources to embrace new platforms, in particular high-performance computers (HPC). Specifically, we discuss the experience of integrating PanDA WMS with a US DOE leadership machine (Titan) to reach sustained production scales of approximately $51 \mathrm{M}$ core-hours a year.

In doing so, we demonstrate how Titan is more efficiently utilized by the mixing of small and short-lived tasks in backfill with regular payloads. Cycles otherwise unusable (or very difficult to use) are used for science, thus increasing the overall utilization on Titan without loss of overall quality-ofservice. The conventional mix of jobs at OLCF cannot be effectively backfilled because of size, duration, and scheduling policies. Our approach is extensible to any HPC with "capability scheduling" policies. We also investigate the use of a pilotabstraction based task execution runtime system to flexibly execute ATLAS and other heterogeneous workloads (molecular dynamics) using regular queues. As such, our approach provides a general solution and investigation of the convergence 
of HPC and HTC execution of workloads.

This work demonstrates a viable production route to delivering large amounts of computing resources to ATLAS and, in the future, to other experimental and observational use cases. This broadens the use of leadership computing while demonstrating how distributed workflows can be integrated with leadership resources, and effectively accommodating HTC and HPC workloads simultaneously.

This paper also provides: (i) a critical evaluation of the many design and operational considerations that have been taken to support the sustained, scalable and production usage of Titan for historically high-throughput workloads, and (ii) early lessons and guidance on designing the next generation of online analytical platforms [5], so that experimental and observational systems can be integrated with production supercomputers in a general and extensible manner.

\section{PANDA OVERVIEW}

PanDA is a Workload Management System (WMS) designed to support the execution of distributed workloads and workflows via pilots [6]. Pilot-capable WMS enable high throughput execution of tasks via multi-level scheduling while supporting interoperability across multiple sites. This is particularly relevant for LHC experiments, where millions of tasks are executed across multiple sites every month, analyzing and producing petabytes of data.

The implementation of PanDA WMS consists of several interconnected subsystems, communicating via dedicated API or HTTP messaging, and implemented by one or more modules. Databases are used to store stateful entities like tasks, jobs and input/output data, and to store information about sites, resources, logs, and accounting.

Currently, PanDA's architecture has five main subsystems: PanDA Server [7], AutoPyFactory [8], PanDA Pilot [9], JEDI [10], and PanDA Monitoring [11]. Other subsystems are used by some of ATLAS workflows but we do not discuss them as they are not relevant to an understanding of how PanDA has been ported to supercomputers. For a full list of subsystems see Ref. [12]. Fig. 1] shows a diagrammatic representation of PanDA main subsystems, highlighting the execution process of tasks while omitting monitoring details to improve readability.

Users submit task descriptions to JEDI (Fig. 1:1) that stores them into a queue implemented by a database (Fig. 112). Tasks are partitioned into jobs of different size, depending on both static and dynamic information about available resources (Fig. 133). Jobs are bound to sites with resources that best match jobs' requirements, and submitted to the PanDA Server for execution (Fig. 114).

Once submitted to the PanDA Server, jobs are stored by the Task Buffer component into a global queue implemented as a database (Fig. 1.5). When jobs are submitted directly to the PanDA Server, the Brokerage component is used to bind jobs to available sites, depending on static information about the resources available for each site. Jobs submitted by JEDI are already bound to sites so no further brokerage is needed.
Once jobs are bound to sites, the Brokerage module communicates to the Data Service module what data sets need to be made available on what site (Fig. 116). The Data Service communicates these requirements to the ATLAS DDM (Fig. 117) that, when needed, replicates data sets on the required sites (Fig. 118).

Meanwhile, AutoPyFactory defines PanDA Pilots, submitting them to a Condor-G agent (Fig. 19). Condor-G schedules these pilots wrapped as jobs to the required sites (Fig. 11 10).

When a PanDA Pilot becomes available, it requests the Job Dispatcher module of the PanDA Server for a job to execute (Fig. 1:11). The Job Dispatcher interrogates the Task Buffer module for a job that is bound to the site of that pilot and ready to be executed. Task Buffer checks the global queue (i.e., the PanDA DB) and, upon availability, returns a job to the Job Dispatcher. The Job Dispatcher dispatches that job to the PanDA Pilot (Fig. 11.12).

Each PanDA Pilot starts a monitoring process on receiving a job and forks a subprocess to execute the job's payload. Input data are transferred from the stage-in location (Fig. 11 13), the job's payload is executed (Fig. 11:14) and once completed, output is transferred to the staging-out location (Fig. 11. 15).

The Data Service module of the PanDA Server tracks and collects the output generated by each job (Fig. 11.16), updating jobs' attributes via the Task Buffer module (Fig. 11 17). When the output of all the jobs of a task are retrieved, it is made available to the user via PanDA Server. When a task is submitted to JEDI, task is instead marked as done (Fig. 1:18) and the result of its execution is made available to the user by JEDI (Fig. 1, 19).

\section{DEPLOYING PANDA ON TITAN}

The upcoming LHC Run 3 will require more resources than the Worldwide LHC Computing Grid (WLCG) can provide. Currently, PanDA WMS uses more than 600,000 cores at more than 100 Grid sites, with an aggregated performance of 8 petaFLOPS. This capacity will be sufficient for the planned analysis and data processing, but it will be insufficient for the Monte Carlo production workflow and any extra activity. To alleviate these challenges, ATLAS is expanding the current computing model to include additional resources such as the opportunistic use of supercomputers.

PanDA WMS has been designed to support distributed Grid computing. Executing ATLAS workloads or workflows involves concurrent and/or sequential runs of possibly large number of jobs, each requiring minimal, if any parallelization and no runtime communication. Thus, computing infrastructure like WLCG have been designed to aggregate large amount of computing resources across multiple sites. While each site may deploy MPI capabilities, usually these are not used to perform distributed computations.

Currently, ATLAS workloads do not require fast interconnects or specilized co-processors, but supercomputers tend not to reach $100 \%$ utilization due to the scheduling of jobs requiring large amount of resources. This offers the possibility to 


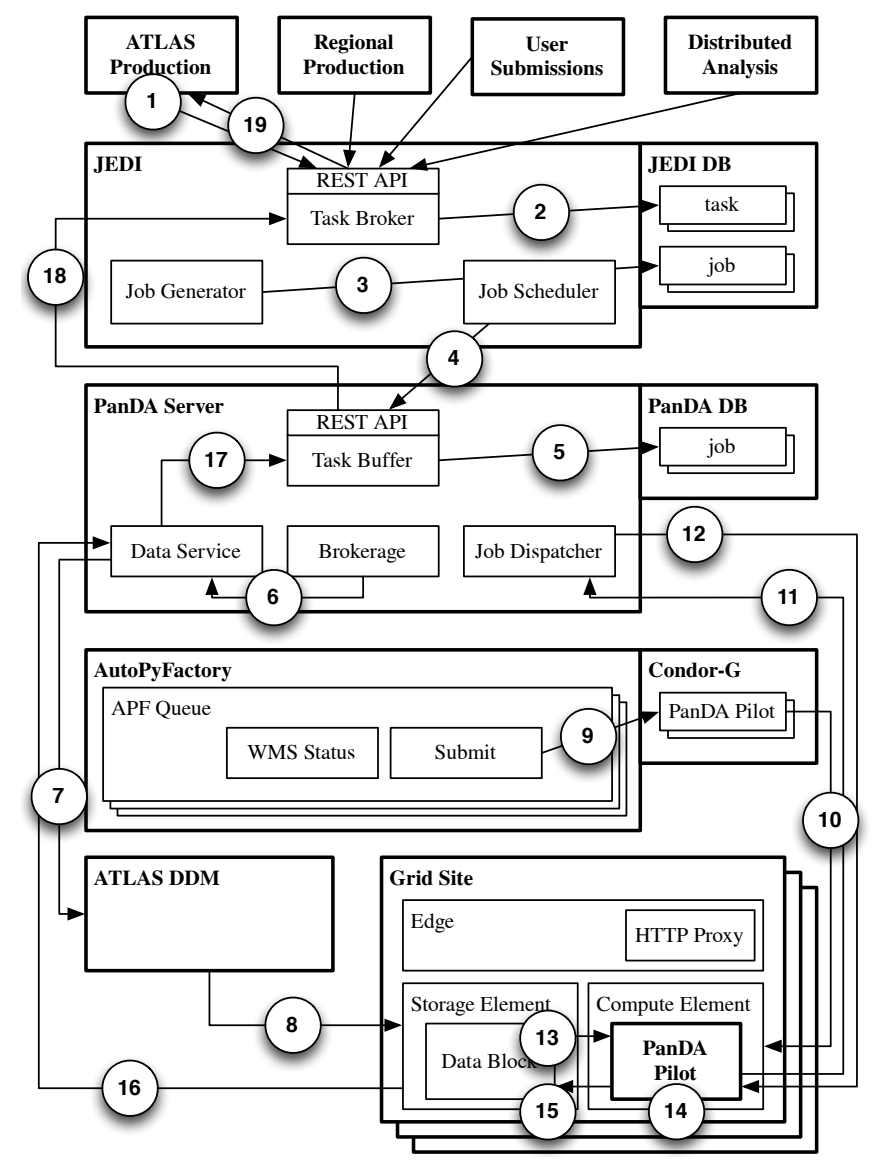

Fig. 1. PanDA WMS architecture. Numbers indicates the JEDI-based execution process described in Several subsystems, components, and architectural and communication details are abstracted to improve clarity.

execute ATLAS-like workloads on supercomputers to increase utilization and reducing the waste of available resources.

We developed a single-point solution to better understand the problem space of enabling a WMS designed for HTC to execute production workflows on resources designed to support HPC. The PanDA team developed a job broker to support the execution of part of the ATLAS production Monte Carlo workflow on Titan, a leadership-class supercomputer managed by the Oak Ridge Leadership Computing Facility (OLCF).

\section{A. Architectures, Interfaces and Workloads}

Titan's architecture, configuration and policies poses several challenges to the deployment of PanDA. The default deployment model of PanDA Pilot is unfeasible on Titan: PanDA Pilot is required to contact the Job Dispatcher of the PanDA Server to pull jobs to execute, but this is not possible on Titan because worker nodes do not offer outbound network connectivity. Further, Titan does not support PanDA's security model based on certificates and virtual organizations, making PanDA's approach to identity management unfeasible. While Titan's data transfer nodes (DTNs) offer wide area network data transfer, an integration with ATLAS DDM is beyond the functional and administrative scope of the current prototyping phase. Finally, the specific characteristics of the execution environment, especially the absence of local storage on the worker nodes and modules tailored to Compute Node Linux, require re-engineering of ATLAS application frameworks.

Currently, very few HEP applications can benefit from Titan's GPUs but some computationally-intensive and non memory-intensive tasks of ATLAS workflows can be offloaded from the Grid to Titan. Further, when HEP tasks can be partitioned into independent jobs, Titan worker nodes can be used to execute up to 16 concurrent payloads, one per each available core. Given these constraints and challenges, the Monte Carlo detector simulation task is most suitable for execution on Titan at the moment. This type of task is mostly computational-intensive, requiring less than 2GB of RAM at runtime and small input data. Detector simulation tasks in ATLAS are performed via AthenaMP [13], the ATLAS software framework integrating the GEANT4 detector simulation toolkit [14]. These tasks account for $\approx 60 \%$ of all the jobs on WLCG, making them a primary candidate for offloading.

Detector simulation is part of the ATLAS production Monte Carlo (MC) workflow [15]. The MC workflow consists of four main stages: event generation, detector simulation, digitization, and reconstruction. Event generation creates sets of particle four-momenta via different generators, e.g., PYTHIA, HERWIG, and many others. Geant4 simulates the ATLAS detector and the interaction between the detector and particles. Each interaction creates a so-called hit and all hits are collected and passed on for digitalization, where hits are further processed to mimic the readout of the detector. Finally, reconstruction operates local pattern recognition, creating high-level objects like particles and jets.

\section{B. PanDA Broker}

The lack of wide area network connectivity on Titan's worker nodes is the most relevant challenge for integrating PanDA WMS and Titan. Without connectivity, Panda Pilots cannot be scheduled on worker nodes because they would not be able to communicate with PanDA Server and therefore pull and execute jobs. This makes impossible to port PanDA Pilot to Titan while maintaining the defining feature of the pilot abstraction: decoupling resource acquisition from workload execution via multi-stage scheduling.

The unavailability of pilots is a potential drawback when executing distributed workloads like MC detector simulation. Pilots are used to increase the throughput of distributed workloads: while pilots have to wait in the supercomputer's queue, once scheduled, they can pull and execute jobs independent from the system's queue. Jobs can be concurrently executed on every core available to the pilot, and multiple generations of concurrent executions can be performed until the pilot's walltime is exhausted. This is particularly relevant for machines like Titan where queue policies privilege parallel jobs on the base of the number of worker nodes they request: the higher the number of nodes, the shorter the amount of queue time (modulo fair-share and allocation policies).

The backfill optimization of Titan's Moab scheduler allows to avoid the overhead of queue wait times without using pi- 
lot abstraction [16]. With this optimization, Moab starts lowpriority jobs when they do not delay higher priority jobs, independent of whether the low-priority jobs were queued after the high-priority ones.

When the backfill optimization is enabled, users can interrogate Moab about the number of worker nodes and walltime that would be available to a low-priority job at that moment in time. If a job is immediately submitted to Titan with that number of worker nodes and walltime, chances are that Moab will immediately schedule it, reducing its queue time to a minimum. In this paper, we call this number of worker nodes and walltime an available 'backfill slot'.

Compared to pilots, backfill has the disadvantage of limiting the amount of resources that can be requested. Pilots are normal jobs: they can request as many worker nodes and walltime as a queue can offer. On the contrary, jobs sized according to an available backfill slot depend on the number of worker nodes and walltime that cannot be given to any other job at that moment in time.

At any point in time, the size of an available backfill slot is typically a small fraction of the total capacity of a resource. Notwithstanding, given the size of Titan this translates into a substantial capacity. Every year, about $10 \%$ of Titan's capacity remains unused [17], corresponding to an average of 30,000 unused cores (excluding GPU cores). This equals to roughly $5 \%$ of the overall capacity of WLCG.

Given the communication requirements of PanDA Pilots and the unused capacity of Titan, PanDA pilot was repurposed to serve as a job broker on the DTN nodes of Titan (Fig. 2). This prototype called 'PanDA Broker' maintains the core modules of PanDA Pilot and its stand-alone architecture. This imposes functional trade-offs (e.g., single-threaded architecture, single MPI PBS script submission) but allows for rapid adoption and iterative optimization. PanDA Brokers are deployed on DTNs because these nodes are part of the OLCF infrastructure and can access Titan without RSA SecureID authentication. DTNs are not part of Titan's worker nodes and, therefore, are not used to execute Titan's jobs.

Currently, up to 20 PanDA Brokers operate within the existing ATLAS production software infrastructure, each supporting the execution of MC detector simulations in 9 steps. Each broker queries the PanDA Server for ATLAS jobs that have been bound to Titan by JEDI (Fig. 2 1). Upon receiving jobs descriptions, PanDA Broker pulls jobs' input files from BNL Data Center to the OLCF Lustre file system (Fig. 22). PanDA Broker queries Titan's Moab scheduler about the current available backfill slot (Fig. 2.3) and creates an MPI script, wrapping enough ATLAS jobs' payload to fit the backfill slot. PanDA Broker submits the MPI script to the Titan's PBS batch system via RADICAL-SAGA (Fig. 204).

Upon execution on the worker node(s) (Fig. 25), the MPI script initializes and configures the execution environment (Fig. 26), and executes one AthenaMP for each available work node (Fig. 277). AthenaMP retrieves events from Lustre (Fig. 2 8) and spawns 1 Geant 4 event simulation process on each of the 16 available cores (Fig. 229). Upon completion

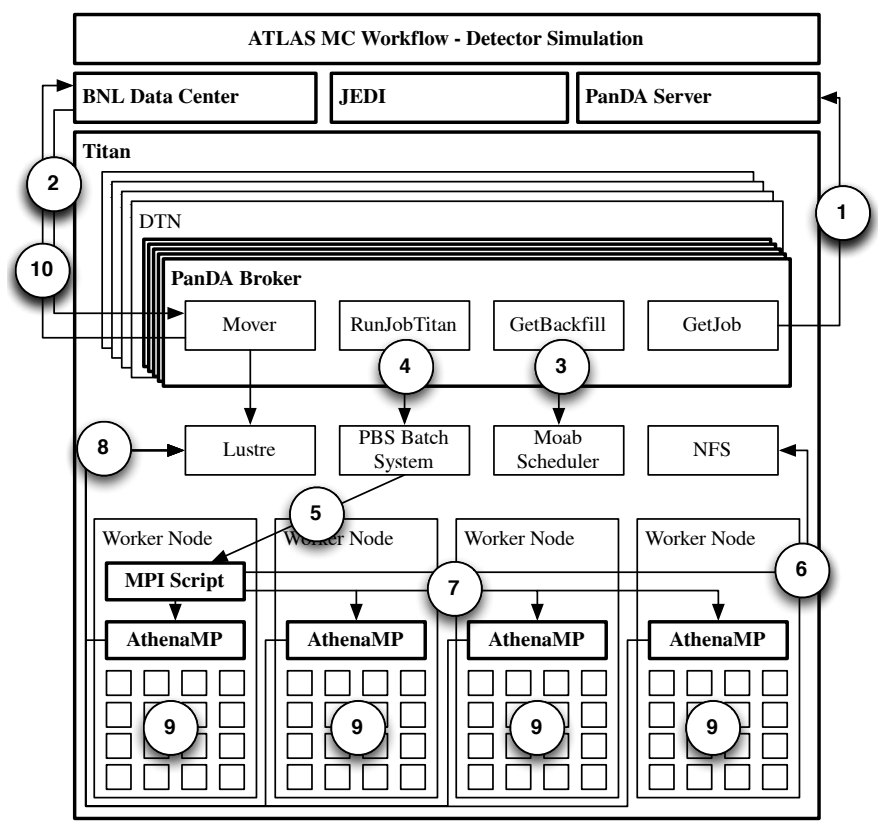

Fig. 2. PanDA Broker architecture as deployed on Titan. Numbers indicates the execution process of a detector simulation job described in

of each MPI script, PanDA Broker transfer the jobs' output to BNL (Fig. 2:10), and performs cleanup.

PanDA Broker implementation is resource specific but the ATLAS team has ported it to other supercomputers, including the HPC2 at the National Research Center "Kurchatov Institute" (NRC-KI) [18], Edison/Cori at the National Energy Research Scientific Computing Center (NERSC) [19], and SuperMUC at the Leibniz Supercomputing Centre (LRZ) [19].

\section{AnAlysis And Discussion}

Currently 20 instances of the PanDA Broker are deployed on 4 DTNs, with 5 instances per DTN. Each broker submits and manages the execution of 15 to 300 jobs, one job for each Titan worker node, and a theoretical maximum concurrent use of 96,000 cores. Since November 2015, PanDA Brokers have operated only in backfill mode, without a defined time allocation, and running at the lowest priority on Titan. Therefore, ATLAS contributed to an increase of Titan's utilization.

We evaluate the efficiency, scalability and reliability of the deployment of PanDA WMS on Titan by characterizing the behavior of both PanDA Broker and AthenaMP. We discuss challenges and limitations of our approach at multiple levels arising from the specifics of workload, middleware and methods. All the measurements were performed between January 2016 and February 2017, hereafter called 'experiment time window'.

\section{A. Characterizing the PanDA Broker on Titan}

We calculate the total amount of backfill availability over a period of time by: (i) polling the available backfill slots at regular intervals during that time window; (ii) converting the number of worker nodes available and their walltime into core- 


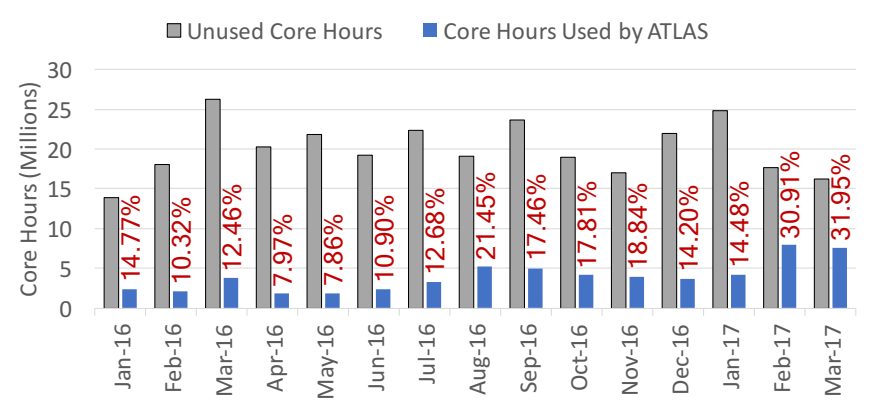

Fig. 3. Titan's total backfill availability: CPU core-hours (gray) and CPU core-hours used by ATLAS (blue). GPU core-hours unaccounted for as they cannot be used by ATLAS. Efficiency of PanDA Brokers defined as percentage of total Titan's backfill availability used by ATLAS (Red labels).

hours; (iii) summing the number of core-hours. We call this number of core-hours 'total backfill availability'.

Fig. 3 shows the total backfill availability on Titan (gray bars) and the number of core-hours of that availability used by ATLAS (blue bars) during the experiment time window. ATLAS consumed a total of $51.4 \mathrm{M}$ core-hours, for an average of $\approx 3.7 \mathrm{M}$ core-hours a month.

PanDA Brokers' efficiency (Fig. 3, red labels) is defined as the fraction (or percentage) of core-hours utilized by the PanDA Brokers of Titan's total backfill availability during the experiment time window. The average efficiency was $18 \%$, with a minimum efficiency of 7.8\% (May 2016) and a maximum efficiency of $30.9 \%$ (Feb. 2017, excluding the preliminary results of March). The total backfill availability was $\approx 21.5 \mathrm{M}$ in April 2016 , and $17.6 \mathrm{M}$ in February 2017. This suggests that the efficiency is invariant of total backfill availability.

During the experiment time window, about $2.25 \mathrm{M}$ detector simulation jobs were completed on Titan, for a total of $225 \mathrm{M}$ events processed. This is equivalent to $0.9 \%$ of all the $250 \mathrm{M}$ detector simulations performed by ATLAS in the same period of time, and $3.5 \%$ of the $6.6 \mathrm{~B}$ events processed by those jobs. These figures confirms the relevance of supercomputers' resource contribution to the LHC Run 2, especially when accounting for the amount of unused total backfill availability and the improvement of PanDA efficiency across the experiment time window.

On February 2017, PanDA Brokers used almost twice as much total backfill availability than in any other month (preliminary results for March 2017 displayed in Fig. 3 confirm this trend). No relevant code update was made during that period and logs indicated that the brokers were able to perform faster. This is likely due to hardware upgrades on the DTNs. The absence of continuous monitoring of those nodes does not allow to quantify bottlenecks but spot measurements of their load indicate that a faster CPU and better networking were likely responsible for the improved performance. Investigations showed an average CPU load of $3.6 \%$ on the upgraded DTNs, as opposed to the "high" utilization reported by OLCF for the previous DTNs. As such, further hardware upgrades seem unlikely to improve significantly the perfor-

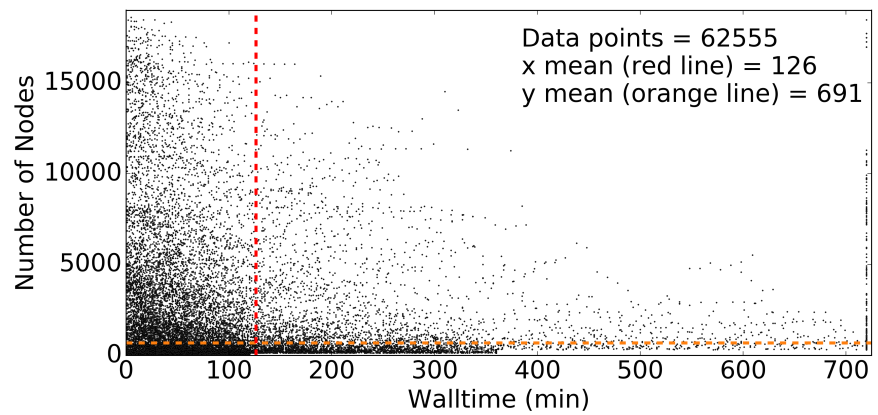

Fig. 4. 62555 measures of Backfill availability on Titan during the experiment time window. Mean number of work nodes available 691; mean walltime available 126 minutes.

mance of PanDA Brokers.

Every detector simulation executed on Titan process 100 events. This number of events is consistent with the physics of the use case and with the average duration of backfill availability. The duration of a detector simulation is a function of the number of events simulated but not all events take the same time to be simulated. One event simulation takes from $\approx 2$ to $\approx 40$ minutes, with a mean of $\approx 14$ minutes. Considering that each worker node process up to 16 events concurrently, 100 events takes an average of 105 minutes to process. As such, PanDA brokers do not use backfill availability with less than 105 minutes walltime.

Fig. 4 shows backfill availability on Titan as a function of number of nodes and the time of their availability (i.e., walltime). We recorded these data by polling Titan's Moab scheduler at regular intervals during the experiment window time. The mean number of nodes was 691 , and their mean walltime was 126 minutes. Detector simulations of 100 events, enable to use down to $5 / 6$ of the mean walltime of backfill availability. As such, it offers a good compromise for PanDA Broker efficiency.

PanDA Broker could fit the number of events to the walltime of each available backfill slot on the base of the distributions of the time taken by one event to be simulated. That specific number of event could then be pulled from the PanDA Event service [20] and given as input to one or more simulations. Once packaged into the MPI script submitted to titan's PBS batch system, these simulations would better fit their available backfill slot, contributing to increase the efficiency of PanDA Brokers.

The transition from a homogeneous to a heterogeneous number of events per detector simulation has implications for the application layer. An even number of events across simulations makes it easier to partition, track and package events across simulations, especially when they are performed on both the Grid and Titan. A homogeneous number of events also helps to keep the size and duration of other stages of the MC workflow (\$II-A) more uniform. Further analysis is needed to evaluate the trade offs between increased efficiency of resource utilization and the complexity that would be introduced at the application layer.

Currently, each PanDA Broker creates, submits, and moni- 
tors a single MPI PBS script at a time. This design is inherited from PanDA Pilot where a single process is spawn at a time to execute the payload. As a consequence, the utilization of a larger portion of Titan's total backfill availability depends on the the number of concurrent PanDA Brokers instantiated on the DTNs: When all the 20 PanDA Brokers have submitted a MPI PBS script, further backfill availability cannot be used.

In August 2016, increasing the number of concurrent PanDA brokers from 4 to 20 markedly improved efficiency (see Fig. 3) but further research is ongoing to understand whether an even greater number of brokers would yield even greater efficiency. This research focuses on evaluating the overheads of input/output files staging, including its impact on DTNs, and on an alternative design of PanDA Broker that enables the concurrent submission of multiple MPI scripts [19]. The understanding will contribute to improving the efficiency of PanDA Brokers beyond the $30 \%$ limit showed in Fig. 3 .

The current design and architecture of the PanDA Broker is proving to be as reliable as PanDA Pilot when used on the WLCG. Between Jan 2016 and Feb 2017, the overall failure rate of all the ATLAS detector simulation jobs was $14 \%$, while the failure rate of jobs submitted to Titan was a comparable 13.6\%. PanDA Brokers were responsible for around the $19 \%$ of the failures, compared to the $29 \%$ of failures produced by the JobDispatcher module of the PanDA Server, and the $13 \%$ failures produced by the Geant4 toolkit.

\section{B. Characterizing the Detector Simulation on Titan}

We use two main parameters to measure the performance of the detector simulation jobs submitted to Titan: (i) the time taken to setup AthenaMP; and (ii) the distribution of the time taken by Geant 4 to simulate a certain number of events.

AthenaMP has an initialization and configuration stage. At initialization time, AthenaMP is assembled from a very large set of shared libraries, depending on the type of payload that will have to be computed. Once initialized, every algorithm and service of AthenaMP is configured via Python scripts. Both these operations result in read operations on the filesystem shared between the worker nodes and the DTNs, including the operations required to access small python scripts.

Initially, all the shared libraries and the python scripts of AthenaMP were stored on the OLCF Spider 2 Lustre file system. However, the I/O patterns of the initialization and configuration stages degraded the performance of the filesystem. This was addressed by moving the AthenaMP distribution to a read-only NFS directory, shared among Titan's DTNs and worker nodes. NFS eliminated the problem of metadata contention, improving metadata read performance from a worse case scenario of 6,300 s on Lustre to $\approx 225$ s on NFS. While these figures are specific to OLCF and Titan, reengineering of AthenaMP could improve startup time on every platform.

Once initialized and configured, AthenaMP is used to execute 16 concurrent Geant4 simulators on a Titan's worker node. Geant4 requires to read events descriptions from a filesystem and simulate them as they would happen within the ATLAS detector. We characterized both the compute performance of the simulation and the impact of acquiring event descriptions on the filesystem.

The AMD Opteron 6274 CPU used on Titan has 16 cores, divided into 8 compute units. Each compute units has 1 floating point (FP) scheduler shared between 2 cores. When using 16 cores for FP-intensive calculations, each pair of cores competes for a single FP scheduler. This creates the overhead shown in Fig. 55 the mean runtime per event for 8 concurrent simulations computing 50 events is 10.8 minutes, while for 16 simulations is 14.25 minutes (consistent with the measured distribution of the duration of event simulation). Despite an inefficiency of almost 30\%, Titan's allocation policy based on number of worker nodes used instead of number of cores does not justify the use of $1 / 2$ of the cores available.

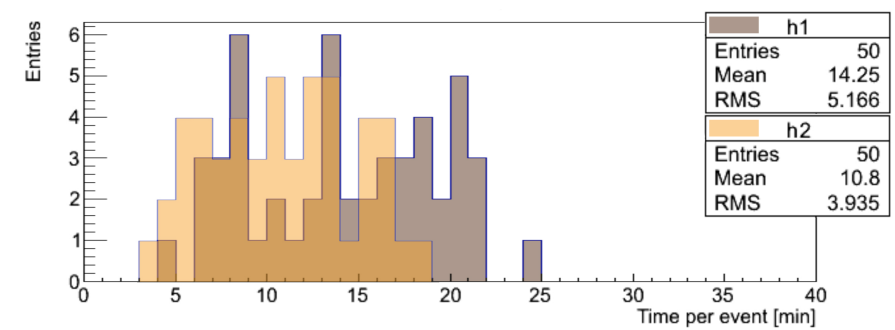

Fig. 5. Distributions of the time taken to simulate one event when placing 2 simulations (h1) or 1 simulation (h2) per Titan's CPU. 2 simulation use 16 cores per node, 1 simulation 8. 50 Events; 1 Titan worker nodes; 16 work threads per node; 100 events per node.

A performance analysis of Titan's AMD CPUs for detector simulations also helps to compare Titan and Grid site performance. Usually, Grid sites expose resources with heterogeneous CPU architectures and 8 (virtual) cores, while Titan's offer an homogeneous 16 cores architecture. We used the rate of events processes per minute as a measure of the efficiency of executing the same detector simulation on Titan or Grid sites. Comparisons of the efficiencies of Titan to the BNL and SIGNET Grid sites, normalized for 8 cores, show that the effective performance per-core at Titan is $\approx 0.57$ event per minute, roughly $1 / 2$ of BNL and $1 / 3$ of SIGNET.

The differences in performance between Titan and the BNL and SIGNET Grid sites are due to the FP scheduler competition but mainly to the availability of newer processors. The heterogeneity of the Grid sites' CPUs explain the higher performance variance we observed compared to the performance consistency we measured on Titan. This difference in performance is compensated by the amount of resources available on Titan (capable of executing up to $30 \%$ /year of the ATLAS detector simulations) and by the lack of further resources available to WLCG. Also, it should be noted that Titan is at the end of its life-cycle and that Summit, Titan's successor, will offer cutting-edge performances.

We studied the impact of acquiring event descriptions on Lustre by analyzing 1,175 jobs ran on the week of 10/25/2016, for a total of 174 hours. Table I shows the overall statistical breakdown of the observed file I/O. ATLAS used between 1 and 300 worker nodes, and 35 on average. $75 \%$ of the jobs run 
by ATLAS consumed less than 25 nodes and $92 \%$ less than 100. During the 174 hours of data collection, 6.75 ATLAS jobs were executed on average per hour, each job running for an average of 1.74 hours. Every job read less than $250 \mathrm{~GB}$ and wrote less than $75 \mathrm{~GB}$ of data and, on average, each job read $20 \mathrm{~GB}$ and wrote $6 \mathrm{~GB}$ of data.

ATLAS jobs are read heavy: On average, the amount of data read per worker node is less than $400 \mathrm{MB}$, while the amount of data written is less than $170 \mathrm{MB}$. Distributions of read and written data are different: The read operation distribution per job shows a long tail, ranging from $12.5 \mathrm{~GB}$ to $250 \mathrm{~GB}$, while the written amount of data has a very narrow distribution.

The metadata I/O breakdown shows that ATLAS jobs yield 23 file open() operations per second (not including file stat() operations) and 5 file close() operations per second, with similar distributions. On average, the maximum number of file open () operations per job is $\approx 170 / \mathrm{s}$ and the maximum number of file close () operations is $\approx 39 / \mathrm{s}$. For the 1,175 ATLAS jobs observed, the total number of file open () operations is $172,089,760$ and the total number of file close() operations is $40,132,992$. The difference between these two values is under investigation: a possible explanation is that ATLAS jobs don't call a file close() operation for every file open() issued.

Overall, the average time taken to read events from input files stored on Lustre is 1,320, comparable to the time taken to read the file required by assembling AthenaMP from NFS. Preliminary investigation shows that this time could be reduced to 40 seconds by loading the event descriptions into the RAM disk available on each worker node. Events descriptions could be transferred from Lustre to the RAM disk while configuring and initializing AthenaMP, almost halving the time currently required by initiating a Geant 4 simulation.

\section{PANDA: The Next Generation Executor}

As explained in III, PanDA Broker was designed to maximize code reutilization of PanDA Pilot. This allowed for rapid adoption and incremental optimization while enabling the parallel development of a more general solution for executing ATALS workloads on HPC resources.

The lack of pilot capabilities in PanDA Broker impacts both the efficiency and the flexibility of PanDA's execution process. Pilots could improve efficiency by increasing throughput and enabling greater backfill utilization. Further, pilots would make it easier to support heterogeneous workloads.

The absence of pilots makes the scheduling of multiple generations of workload on the same PBS job impossible: once a statically defined number of detector simulations are packaged into a PBS job and this job is queued on Titan, no further simulations can be added to that job. New simulations have to be packaged into a new PBS job that needs to be submitted to Titan based upon backfill and PanDA Brokers availability.

The support of multiple generations of workload would enable more efficient use of the backfill availability walltime. Currently, when a set of simulations ends, the PBS job also ends, independent of whether more wall-time would still be available. With a pilot, additional simulations could be executed to utilize all the available wall-time, while avoiding further job packaging and submission overheads.

Multiple generations would also relax two assumptions of the current execution model: knowing the number of simulations before submitting the MPI script, and having a fixed number of events per simulation (currently 100). Pilots would enable the scheduling of simulations independently from whether they were available at the moment of submitting the pilot. Further, simulations with a varying number of events could be scheduled on a pilot, depending on the amount of remaining walltime and the distribution of execution time per event, as shown in $\$$ III-B. Fig. 5. These capabilities would increase the efficiency of the PanDA Broker when there is a large difference between the number of cores and walltime.

Pilots can offer a payload-independent scheduling interface while hiding the mechanics of coordination and communication among multiple worker nodes. This could eliminate the need for packaging payload into MPI scripts within the broker, greatly simplifying the submission process. This simplification would also enable the submission of different types of payload, without having to develop a specific PBS script for each payload. The submission process would also be MPIindependent, as MPI is used for coordination among multiple worker nodes, not by the payload.

\section{A. Implementation}

The implementation of pilot capabilities within the PanDA Broker require quantification of the effective benefits that it could yield and, on the base of this analysis, a dedicated engineering effort. We developed a prototype of a pilot system capable of executing on Titan to study experimentally the quantitative and qualitative benefits that it could bring to PanDA. We called this prototype Next Generation Executor (NGE).

NGE is a runtime system to execute heterogeneous and dynamically determined tasks that constitute workloads. Fig. 6 illustrates its current architecture as deployed on Titan: the two management modules (Pilot and Unit) represent a simplified version of the PanDA Broker while the agent module is the pilot submitted to Titan and executed on its worker nodes. The communication between PanDA Broker and Server is abstracted away as it is not immediately useful to evaluate the performance and capabilities of a pilot on Titan.

NGE exposes an API to describe workloads (Fig. 6, green squares) and pilots (Fig. 6, red circles), and to instantiate a PilotManager and a UnitManager. The PilotManager submits pilots to Titan's PBS batch system via SAGA API (Fig. 6 dash arrow). Once scheduled, the Pilot Agent is bootstrapped on a MOM node and the Agent's Executors on worker nodes.

The UnitManager and the Pilot Agent communicate via a database instantiated on a DTN so as to be reachable by both modules. The UnitManager schedules units to the Agent's Scheduler (Fig. 6, solid arrow) and the Agent's Scheduler schedules the units on one or more Agent's Executor.

The Pilot Agent uses the Open Run-Time Environment (ORTE) for communication and coordination of the execu- 


$\begin{array}{lllllllll} & \text { Num. Nodes } & \text { Duration (s) } & \text { Read (GB) } & \text { Written (GB) } & \text { GB Read/nodes } & \text { GB Written/nodes } & \text { open }() & \text { close }() \\ \text { Min } & 1 & 1,932 & 0.01 & 0.03 & 0.00037 & 0.02485 & 1,368 & 349 \\ \text { Max } & 300 & 7,452 & 241.06 & 71.71 & 0.81670 & 0.23903 & 1,260,185 & 294,908 \\ \text { Average } & 35.66 & 6,280.82 & 20.36 & 6.87 & 0.38354 & 0.16794 & 146,459.37 & 34,155.74 \\ \text { Std. Dev. } & 55.33 & 520.99 & 43.90 & 12.33 & 0.19379 & 0.03376 & 231,346.55 & 53,799.08\end{array}$

THE STATISTICAL BREAKDOWN OF THE I/O IMPACT OF 1,175 JOBS ATLAS EXECUTED AT OLCF FOR THE WEEK OF 10/25/16

\begin{tabular}{|c|c|c|c|c|c|c|}
\hline & & & $\square \square$ & 20000 & & Application \\
\hline & & & $\begin{array}{ll}1 \\
1\end{array}$ & & Pilot API & \\
\hline $\begin{array}{r}\text { Pilc } \\
\text { Mana }\end{array}$ & & $\begin{array}{l}\text { Pilot } \\
\text { Launch }\end{array}$ & er $\quad \vdots t$ & $\begin{array}{c}\text { Unit } \\
\text { Scheduler }\end{array}$ & $\begin{array}{c}\text { Unit } \\
\text { Manager }\end{array}$ & DTS \\
\hline & & & & & SAGAAPI & \\
\hline & & & & & & MongoDB \\
\hline Titan & Pilot & Agent & $\begin{array}{l}\text { MOM Scheduler } \\
\text { Node }\end{array}$ & $\begin{array}{l}\text { Worker Executor } \\
\text { Node }\end{array}$ & $\begin{array}{l}\text { Worker } \\
\text { Node }\end{array}$ & $\begin{array}{l}\text { Executor } \\
0\end{array}$ \\
\hline
\end{tabular}

Fig. 6. NGE Architecture: The PilotManager and UnitManager reside on a DTN while the Pilot Agent is executed on a worker node. Color coding: gray for entities external to NGE; white for APIs; purple for NGE's modules; green for pilots; yellow for module's components.

tion of units. This environment is a critical component of the OpenMPI implementation [21]. ORTE is able to minimize the system overhead while submitting tasks by avoiding filesystem bottlenecks and race conditions with network sockets.

\section{B. Experiments}

We designed experiments to characterize the performance of the NGE on Titan, with an emphasis on understanding its overhead and thus the cost of introducing new functionalities. We perform three groups of experiments in which we investigate the weak scalability, weak scalability with multiple generation, and strong scalability of the NGE.

Each experiment entails executing multiple instances of AthenaMP to simulate a pre-determined number of events. All the experiments have been performed by configuring AthenaMP to use all the 16 cores of Titan's worker nodes.

We measured the execution time of the pilots and of AthenaMP within them, collecting timestamps at all stages of the execution. Experiments were performed by submitting pilots to Titan's batch queue. The turnaround time of an individual run is determined by queue waiting times. Since we are interested only in the performances of the NGE, we removed queue time from our statistics.

1) Weak scalability: In this experiment we run as many AthenaMP instances (hereafter referred to as tasks) as the number of nodes controlled by the pilot. Each AthenaMP simulates 100 events, requiring $\sim 4200$ seconds on average.

Tasks do not wait within the Agent's queue since one node is available to each AthenaMP instance. Task execution overheads result primarily from three factors: (i) the initial bootstrapping of the pilot on the nodes; (ii) the UnitManager's dispatching of units (tasks) to the agent; and (iii) time for the Agent to bootstrap all the tasks on the nodes.

We tested pilots with 250, 500, 1000 and 2000 worker nodes and 2 hours walltime. The time duration is determined by

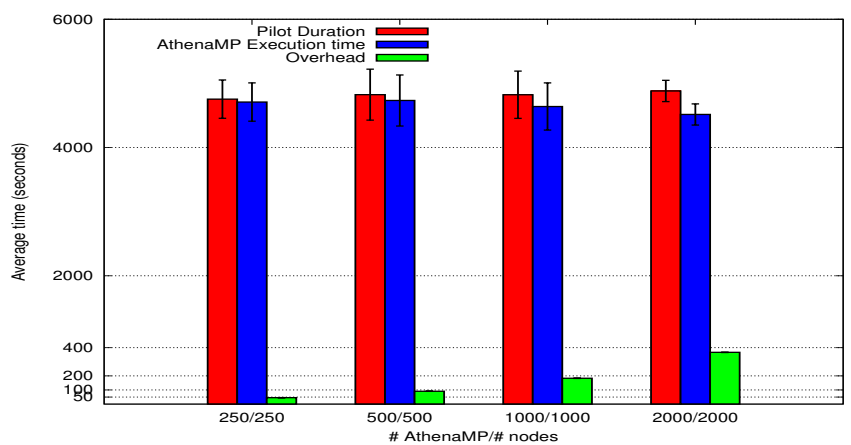

Fig. 7. Weak scalability: average pilot duration, average duration of one AthenaMP execution, and pilot's overhead as a function of pilot sizes (200, 500,1000 and 2000 nodes).

the Titan's walltime policy. Fig. 7 depicts the average pilot duration, the average execution time of AthenaMP, and the pilot overhead as function of the pilot size.

We observe that, despite some fluctuations due to external factors (e.g., Titan's shared filesystem and the shared database used by the NGE), the average execution time of AthenaMP ranges between 4500 and 4800 seconds. We also observe that in all the cases the gap between AthenaMP execution times and the pilot durations is minimal, although it slightly increases with the pilot size. We notice that NGE's overhead grows linearly with the number of units.

2) Weak scalability with multiple generation: The NGE provides an important new capability of submitting multiple generations of tasks to the same pilot. In order to investigate the cost of doing so, we performed a variant of the weak scalability experiments. This stresses the pilot's components, as new tasks are scheduled for execution on the Agent while other tasks are still running.

In these experiments, we run five AthenaMP instances per node. As these experiments are designed to investigate the overhead of scheduling and bootstraping of AthenaMP instances, the number of events simulated by each AthenaMP task was reduced to sixteen such that the running time of each AthenaMP was $\approx 1,200$ seconds on average. This experiment design choice does not affect the objectives or accuracy of the experiments, but allows us to scale experiments to large node counts by conserving project allocation.

We ran pilots with 256, 512, 1024 and 2048 worker nodes and 3 hours walltime. Fig. 8 depicts the average pilot duration, the average execution time of five generations of AthenaMP, and the corresponding overhead. The difference between the two durations is more marked than in the previous experiments. Despite this, we notice that the growth of the overhead 


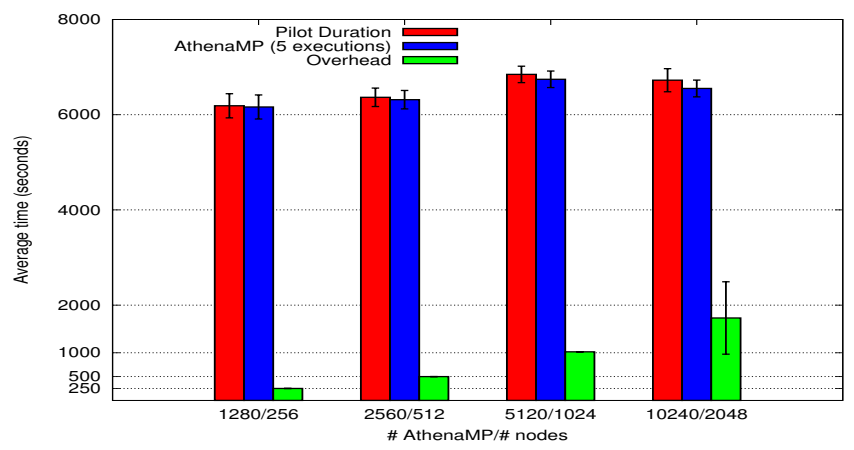

Fig. 8. Weak scalability with multiple generations (where each generation has approximately 1/6th the number of events compared to Fig. 7): average pilot duration, average duration of sequential AthenaMP executions, and pilot's overhead for pilot with 256, 512, 1024 and 2048 nodes.

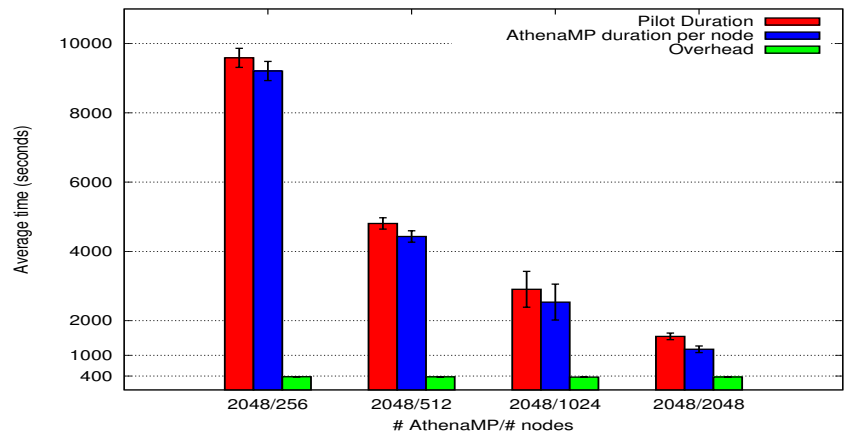

Fig. 9. Strong scalability: Average pilot duration, average duration of sequential AthenaMP executions, and pilot's overhead for pilots with 256, 512, 1024 and 2048 nodes.

is consistent with the increment of the number of tasks per node for pilots with 256, 512 and 1024 worker nodes, and less than linear for the pilot with 2048 worker nodes.

3) Strong scalability: We investigate strong scalability by running the same number of tasks for different pilot sizes. We used 2048 AthenaMP instances and pilots with 256, 512, 1024 and 2048 nodes. Thus, the number of AthenaMP instances per node (i.e., generations) is eight for the smallest pilot (256 nodes), one for the largest pilot (2048 nodes), and with the number of generations of AthenaMP decreasing with the pilot size. These experiments are designed to investigate whether pilot overhead is affected by the degree of concurrency within the pilot and/or the number of tasks. Each AthenaMP instance simulates sixteen events as in the previous experiment.

Fig. 9 shows the average pilot duration and the average execution time of possibly sequential AthenaMP instances. We notice that the difference between the pilot duration and the AthenaMP execution times is almost constant for all the pilot sizes, although the overall duration of the pilot decreases linearly with the pilot size.

\section{RELATED WORK}

Several pilot-enabled WMS were developed for the LHC experiments: AliEn [22] for ALICE; DIRAC [23] for LHCb; GlideinWMS [24] for CMS; and PanDA [25] for ATLAS. These systems implement similar design and architectural principles: centralization of task and resource management, and of monitoring and accounting; distribution of task execution across multiple sites; unification of the application interface; hiding of resource heterogeneity; and collection of static and sometimes dynamic information about resources.

AliEn, DIRAC, GlideinWMS and PanDA all share a similar design with two types of components: the management ones facing the application layer and centralizing the capabilities required to acquire tasks' descriptions and matching them to resource capabilities; and resource components used to acquire compute and data resources and information about their capabilities. Architecturally, the management components include one or more queue and a scheduler that coordinates with the resource modules via push/pull protocols. All resource components include middleware-specific APIs to request for resources, and a pilot capable of pulling tasks from the management modules and executing them on its resources.

AliEn, DIRAC, GlideinWMS and PanDA also have similar implementations. These WMS were initially implemented to use Grid resources using the Condor software ecosystem [2]. Accordingly, all LHC WMS implemented Grid-like authentication and authorization systems and adopted a computational model based on distributing a large amount of single/few-cores tasks across hundreds of sites.

All LHC experiments produce and process large amounts of data from actual collisions in the accelerator and from their simulations. Dedicated, multi-tiered data systems have been built to store, replicate, and distributed these data. All LHC WMS interface with these systems to move data to the sites where related compute tasks are executed or to schedule compute tasks where (large amount of) data are already stored.

It is interesting to note that most WMS developed to support LHC experiments are gradually evolving towards integrating HPC resources, though none have reached sustained operational usage at the scales that PanDA has achieved for ATLAS on Titan.

\section{CONCLUSION}

The deployment of PanDA Broker on Titan enabled distributed computing on a leadership-class HPC machine at unprecedented scale. In the past 13 months, PanDA WMS has consumed almost 52M core-hours on Titan, simulating $3.5 \%$ of the total number of detector events of the ATLAS production Monte Carlo workflow. We described the implementation and execution process of PanDA WMS ( $\mathrm{II}$ ) and PanDA Broker ( III), showing how they support and enable distributed computing at this scale on Titan, a leadership-class HPC machine managed by OCLF.

We characterized the efficiency, scalability and reliability of both PanDA Broker and AthenaMP as deployed on Ti$\tan ($ III). Our characterization highlighted the strengths and limitations of the current design and implementation: PanDA Brokers enable the sustained execution of millions of simulations per week but further work is required to optimize its efficiency and reliability (IV-A). PanDA Brokers support the concurrent execution of multiple AthenaMP instances, enabling each AthenaMP to perform the concurrent execution 
of up to 16 Geant4 simulators. Nonetheless, our characterization showed how improving I/O performance could reduce overheads ( $\$$ IV-B $)$, increasing the overall utilization of Titan's backfill availability. We introduced PanDA's next generation executor for HPC systems, characterized its ability to support multi-generation workloads and analyzed its scaling behavior. Performance tests on the next generation executor demonstrate linear strong and weak scalability over several orders of magnitude of task and node counts. Qualitatively, it enables the high-performance execution of new workloads and advanced execution modes of traditional workloads.

HEP was amongst the first, if not the first experimental community to realize the importance of using WMS to manage their computational campaign(s). As computing becomes increasingly critical for a range of experiments, the experience foreshadows the importance of WMS for other experiments (such as SKA, LSST etc.). These experiments will have their own workload characteristics, resources types and federation constraints, as well metrics of performance. The experience captured in this paper should be useful for designing WMS for computational campaigns and will provide a baseline to evaluate the relative merits of different approaches.

The 52M core hours used by ATLAS, via PanDA, is over $2 \%$ of the total utilization on Titan over the same period, bringing the time-averaged utilization of Titan to be consistently upwards of $90 \%$. Given that the average utilization of most other leadership class machines is less (e.g., NSF's flagship Blue Waters the average utilization fluctuates between 60 $80 \%$ (see XDMoD[26])) there is ample headroom for similar approaches elsewhere. These unprecedented efficiency gains aside, this work is just a starting point towards more effective operational models for future leadership and online analytical platforms [5]. These platforms will have to support ever increasing complex workloads with varying models for dynamic resource federation.

Acknowledgements: This work is funded by Award number DESC0016280 from the Office of Advanced Scientific Computing Research within the Department of Energy. This research used resources of the Oak Ridge Leadership Computing Facility at the Oak Ridge National Laboratory, which is supported by the Office of Science of the U.S. Department of Energy under Contract No. DE-AC05-00OR22725.

\section{REFERENCES}

[1] I. Foster and C. Kesselman, The Grid 2: Blueprint for a new computing infrastructure. Amsterdam, Netherlands: Elsevier, 2003.

[2] D. Thain, T. Tannenbaum, and M. Livny, "Distributed computing in practice: the Condor experience," Concurrency and computation: practice and experience, vol. 17, no. 2-4, pp. 323-356, 2005.

[3] G. Aad et al., "The ATLAS experiment at the CERN large hadron collider," JINST, vol. 3, p. S08003, 2008.

[4] T. Maeno, "Overview of ATLAS PanDA workload management," $J$. Phys.: Conf. Ser, vol. 331, no. 7, p. 072024, 2011.

[5] A. N. L. Argonne National Laboratory. (2017) Future online analysis platform: Building a research roadmap for future science platforms. [Online]. Available: https://press3.mcs.anl.gov/futureplatform/

[6] M. Turilli, M. Santcroos, and S. Jha, "A comprehensive perspective on pilot-jobs," ACM Computing Surveys (accepted, in press), arXiv preprint arXiv:1508.04180v3, 2017.

[7] T. Maeno, K. De, T. Wenaus, P. Nilsson, G. Stewart, R. Walker, A. Stradling, J. Caballero, M. Potekhin, D. Smith et al., "Overview of ATLAS PanDA workload management," in J. Phys.: Conf. Ser, vol. 331,2011 , p. 072024.
[8] J. Caballero, J. Hover, P. Love, and G. Stewart, "AutoPyFactory: a scalable flexible pilot factory implementation," in J. Phys.: Conf. Ser, vol. 396, 2012, p. 032016

[9] P. Nilsson, J. Caballero, K. De, T. Maeno, A. Stradling, T. Wenaus, A. Collaboration et al., "The ATLAS PanDA pilot in operation," in $J$. Phys.: Conf. Ser., vol. 331, 2011, p. 062040.

[10] M. Borodin, K. De, J. Garcia, D. Golubkov, A. Klimentov, T. Maeno, A. Vaniachine et al., "Scaling up ATLAS production system for the LHC run 2 and beyond: project ProdSys2," in J. Phys.: Conf. Ser., vol. 664, 2015, p. 062005

[11] A. Klimentov, P. Nevski, M. Potekhin, and T. Wenaus, "The ATLAS PanDA monitoring system and its evolution," in J. Phys.: Conf. Ser. vol. 331, 2011, p. 072058.

[12] A. P. Team. (2017) The PanDA production and distributed analysis system. [Online]. Available: https://twiki.cern.ch/twiki/bin/view/PanDA/ PanDA

[13] G. Aad, B. Abbott, J. Abdallah, A. Abdelalim, A. Abdesselam, O. Abdinov, B. Abi, M. Abolins, H. Abramowicz, H. Abreu et al., "The ATLAS simulation infrastructure," The European Physical Journal C, vol. 70, no. 3, pp. 823-874, 2010 .

[14] S. Agostinelli, J. Allison, K. a. Amako, J. Apostolakis, H. Araujo, P. Arce, M. Asai, D. Axen, S. Banerjee, G. Barrand et al., "GEANT4simulation toolkit," Nuclear instruments and methods in physics research section A: Accelerators, Spectrometers, Detectors and Associated Equipment, vol. 506, no. 3, pp. 250-303, 2003.

[15] A. Rimoldi, A. Dell'Acqua, A. di Simone, M. Gallas, A. Nairz, J. Boudreau, V. Tsulaia, and D. Costanzo, "Atlas detector simulation: Status and outlook," in Astroparticle, Particle and Space Physics, Detectors and Medical Physics Applications, vol. 1, 2006, pp. 551-555.

[16] I. Adaptive Computing Enterprises. (2014) Maui scheduler administrator's guide: Backfill. [Online]. Available: http: //docs.adaptivecomputing.com/maui/8.2backfill.php

[17] A. D. Barker, D. E. Bernholdt, A. S. Bland, J. D. Gary, J. J. Hack, S. T. McNally, J. H. Rogers, B. Smith, T. Straatsma, S. R. Sukumar, K. G. Thach, S. Tichenor, S. S. Vazhkudai, and J. C. Wells, "High performance computing facility operational assessment 2015: Oak ridge leadership computing facility," Oak Ridge National Laboratory (ORNL), Oak Ridge, TN (United States). Oak Ridge Leadership Computing Facility (OLCF), Tech. Rep. ORNL/SPR-2016/110, Mar 2016. [Online]. Available: http://www.osti.gov/scitech/servlets/purl/1324094

[18] A. Belyaev, A. Berezhnaya, L. Betev, P. Buncic, K. De, D. Drizhuk, A. Klimentov, Y. Lazin, I. Lyalin, R. Mashinistov et al., "Integration of russian tier-1 grid center with high performance computers at NRC-KI for LHC experiments and beyond HENP," in J. Phys.: Conf. Ser., vol. 664, 2015, p. 092018

[19] F. H. Barreiro Megino, S. Padolski, D. Oleynik, T. Maeno, S. Panitkin, K. De, T. Wenaus, A. Klimentov, and P. Nilsson, "PanDA for ATLAS distributed computing in the next decade," The ATLAS collaboration, Tech. Rep. ATL-COM-SOFT-2016-049, 2016.

[20] P. Calafiura, K. De, W. Guan, T. Maeno, P. Nilsson, D. Oleynik, S. Panitkin, V. Tsulaia, P. Van Gemmeren, and T. Wenaus, "The ATLAS event service: A new approach to event processing," in J. Phys.: Conf. Ser, vol. 664, 2015, p. 062065.

[21] R. H. Castain, T. S. Woodall, D. J. Daniel, J. M. Squyres, B. Barrett, and G. E. Fagg, "The open run-time environment (OpenRTE): A transparent multi-cluster environment for high-performance computing," in Proc. 12th European PVM/MPI Users' Group Meeting, 2005, pp. 225-232.

[22] S. Bagnasco, L. Betev, P. Buncic, F. Carminati, F. Furano, A. Grigoras, C. Grigoras, P. Mendez-Lorenzo, A. J. Peters, and P. Saiz, "The ALICE workload management system: Status before the real data taking," $J$. Phys.: Conf. Ser., vol. 219, p. 062004. 6p, 2010.

[23] S. Paterson, J. Closier, and t. L. D. Team, "Performance of combined production and analysis WMS in DIRAC," J. Phys.: Conf. Ser., vol. 219, no. 7, p. $072015,2010$.

[24] I. Sfiligoi, "glideinwms - a generic pilot-based workload management system," in J. Phys.: Conf. Ser., vol. 119, 2008, p. 062044.

[25] T. Maeno, K. De, A. Klimentov, P. Nilsson, D. Oleynik, S. Panitkin, A. Petrosyan, J. Schovancova, A. Vaniachine, T. Wenaus et al., "Evolution of the ATLAS PanDA workload management system for exascale computational science," in J. Phys.: Conf. Ser., vol. 513, 2014, p. 032062.

[26] X. B. Waters. (2017) Xdmod - blue waters. [Online]. Available: http://xdmod.ncsa.illinois.edu/\#tg_usage:statistic_Jobs_none_utilization 